\title{
Practical synthesis of some novel unsymmetrical 1,3-dialkyl pyrimidine derivatives at room temperature
}

\author{
Ali Khalafi-Nezhad,*a Abdolkarim Zare, ${ }^{a}$ Abolfath Parhami, \\ and Mohammad N. Soltani Rad \\ ${ }^{a}$ Department of Chemistry, College of Sciences, Shiraz University, Shiraz 71454, Iran \\ ${ }^{b}$ Department of Chemistry, College of Basic Sciences, Shiraz University of Technology, Shiraz \\ 71555-313, Iran \\ E-mail: khalafi@chem.susc.ac.ir
}

\begin{abstract}
The efficient synthesis of some novel unsymmetrical 1,3-dialkylpyrimidines is described. N3Alkylation of $N 1$-substituted pyrimidine derivatives with various functionalized alkyl halides in the presence of catalytic amount of tetrabutylammonium bromide (TBAB) and $\mathrm{Cs}_{2} \mathrm{CO}_{3}$ in $\mathrm{MeCN}$ affords the title compounds. The reactions proceed at room temperature and the products are obtained in good to excellent yields.
\end{abstract}

Keywords: 1,3-Dialkylpyrimidine, nucleobase, $N 3$-alkylation, alkyl halide

\section{Introduction}

In recent years, there has been a growing interest in the synthesis of bioactive compounds in organic chemistry. ${ }^{1}$ One important class of these compounds is modified $\mathrm{N}$-substituted nucleobases, including acyclic, ${ }^{2}$ carboacyclic, ${ }^{3}$ carbocyclic, ${ }^{4}$ aza $^{5}$ and thionucleosides. ${ }^{6}$ Some nucleoside derivatives possessing pyrimidine nucleobases have biological activities. The $N 1-\beta-$ hydroxy alkyl pyrimidine nucleobases seem to have attractive structures for investigation of carcinogenesis $^{3 \mathrm{a} a}$ and as accesses to therapeutic agents such as antiviral pyrimidine derivatives. ${ }^{3 \mathrm{~b}}$ Furthermore, the $N 1, N 3$-disubstituted pyrimidines are interesting as these compounds have required scaffold to consider as intercalating and alkylating agents. ${ }^{7}$ The intercalating and alkylating agents nowadays have critical role in cancer chemotherapy. ${ }^{7}$ Antiviral activity has been also reported for these compounds. ${ }^{8} 1,3$-Dialkylpyrimidine derivatives are prepared by $N 3$ alkylation of 1-alkylpyrimidines ${ }^{9}$ or $N 1, N 3$-dialkylation of pyrimidine nucleobases. ${ }^{10}$ For this purpose, several bases, such as sodium hydride, ${ }^{9 a}$ tetrabutylammonium fluoride (TBAF) ${ }^{9 b}$ potassium fluoride, ${ }^{9 \mathrm{c}}$ potassium carbonate, ${ }^{9 \mathrm{~d}}$ magnesium oxide ${ }^{10 \mathrm{a}}$ and potassium hydroxide ${ }^{10 \mathrm{~b}}$ have been used. These methods are often associated with one or more of the following 
drawbacks: (i) the use of DMF as solvent with cumbersome workup of the reaction mixture, ${ }^{9 a, 9 d}$ (ii) long reaction times; ${ }^{9 b, 9 \mathrm{~d}}$ (iii) low yields; $;{ }^{9 \mathrm{~d}, 10}$ and (iv) harsh conditions in which the use of low boiling point alkylating agent is difficult. ${ }^{9 c-d, 10 a}$ To overcome these drawbacks and also in extension of our previous studies on synthesis of nucleoside derivatives, ${ }^{10 a, 11}$ we describe here a practical, simple and efficient synthesis of some novel unsymmetrical 1,3-dialkylpyrimidine derivatives via $N 3$-alkylation of 1-alkylpyrimidines with carbon electrophiles in the presence of catalytic amount of TBAB and $\mathrm{Cs}_{2} \mathrm{CO}_{3}$ in $\mathrm{MeCN}$ at room temperature (Scheme 1). In these mild conditions, alkylating agents with low boiling point, such as allyl bromide and propargyl chloride can be easily employed.

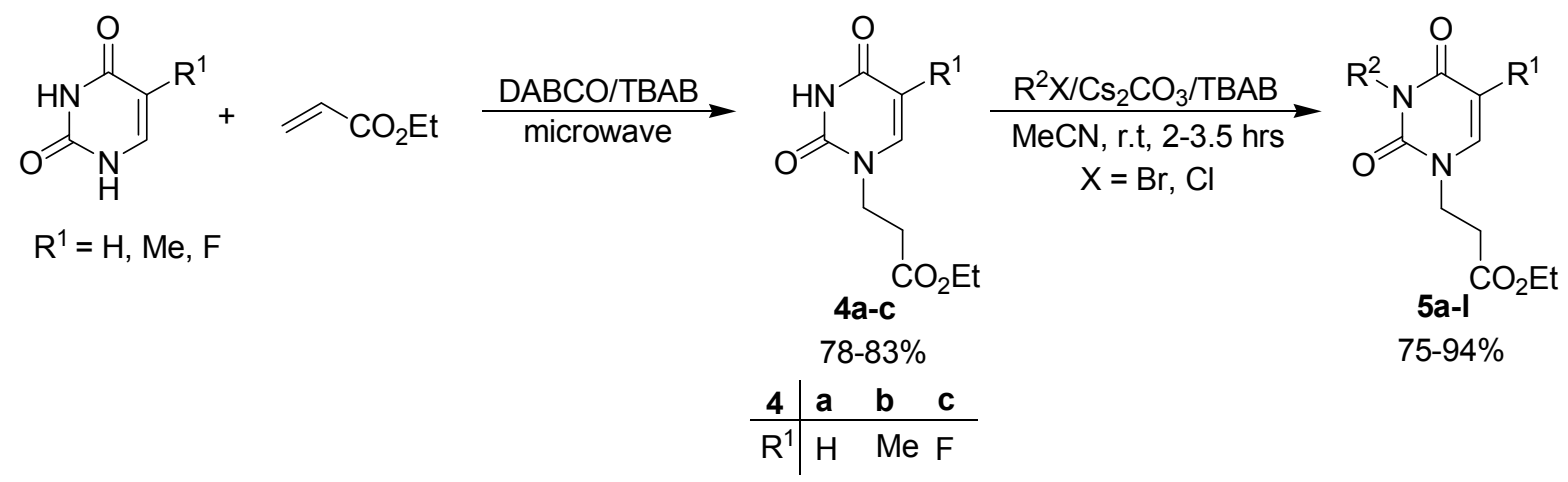

\begin{tabular}{|c|c|c|c|c|c|c|c|c|}
\hline 5 & $\mathrm{R}^{1}$ & $R^{2}$ & 5 & $\overline{R^{1}}$ & $\mathrm{R}^{2}$ & 5 & $\overline{\mathrm{R}^{1}}$ & $\mathrm{R}^{2}$ \\
\hline a & $\mathrm{H}$ & $\mathrm{Cl}_{\mathrm{Cl}}$ & e & $\mathrm{H}$ & $\mathrm{Ol}$ & i & Me & \\
\hline b & $\mathrm{H}$ & $\overbrace{\mathrm{NPht}}$ & f & $\mathrm{H}$ & & j & Me & \\
\hline c & $\mathrm{H}$ & $(x) \sqrt{3} \sim \mathrm{NPht}$ & g & $\mathrm{H}$ & & k & $\mathrm{F}$ & \\
\hline d & $\mathrm{H}$ & $\mathrm{CO}_{2} \mathrm{Et}$ & h & $\mathrm{H}$ & $\equiv$ & I & $\mathrm{F}$ & \\
\hline
\end{tabular}

Scheme 1. Synthesis of $N 1, N 3$-dialkylated pyrimidines.

\section{Results and Discussion}

In order to optimize the reaction conditions, the reaction of carboacyclic nucleoside $4 \mathbf{a}$ with 1bromo-3-chloropropane was studied as a model reaction to provide compound 5a. At first, the influence of various bases in the presence of catalytic amounts of TBAB was examined to evaluate their capabilities (Table 1). Higher yields and shorter reaction times were observed when $\mathrm{Cs}_{2} \mathrm{CO}_{3}$ was used as base. Therefore, $\mathrm{Cs}_{2} \mathrm{CO}_{3}$ was the base of choice for all reactions.

Several reactions were carried out to understand the effect of other solvents beside $\mathrm{MeCN}$ on the results of reaction. A set of anhydrous solvents was examined for the previously described 
model reaction. The results are depicted in Table 2. As Table 2 indicates, the best results were obtained with $\mathrm{MeCN}$.

To compare the efficiency of solution conditions versus solvent-free conditions, we have examined the model reaction under solvent-free conditions (microwave and thermal). The results are summarized in Table 3. As it is clear from Table 3, the solution conditions are more efficient.

Table 1. The influence of bases $(0.010 \mathrm{~mol})$ on reaction of compound $4 \mathrm{a}(0.010 \mathrm{~mol})$ with 1bromo-3-chloropropane $(0.015 \mathrm{~mol})$ in the presence of TBAB $(0.001 \mathrm{~mol})$ in $\mathrm{MeCN}(30 \mathrm{~mL})$ at room temperature

\begin{tabular}{rrcc}
\hline Entry & Base & Time $(h)$ & Yield $^{a}(\%)$ \\
\hline 1 & $\mathrm{Cs}_{2} \mathrm{CO}_{3}$ & 2 & 94 \\
2 & $\mathrm{CsHCO}_{3}$ & 6 & 42 \\
3 & $\mathrm{CsOH}$ & 4 & 30 \\
4 & $\mathrm{NaOH}$ & 6 & 26 \\
5 & $\mathrm{~K}_{2} \mathrm{CO}_{3}$ & 6 & 69 \\
6 & $\mathrm{MgO}$ & 6 & trace \\
7 & $\mathrm{CaO}$ & 6 & trace \\
8 & $t-\mathrm{BuOK}$ & 6 & 33 \\
9 & $\mathrm{DABCO}$ & 6 & 9 \\
\hline
\end{tabular}

${ }^{\mathrm{a}}$ Isolated yield

Table 2. The effect of solvents on reaction in the presence of $T B A B$ and $\mathrm{Cs}_{2} \mathrm{CO}_{3}$ at room temperature

\begin{tabular}{rrcc}
\hline Entry & Solvent & Time $(h)$ & Yield $^{a}(\%)$ \\
\hline 1 & MeCN & 2 & 94 \\
2 & Acetone & 2 & 92 \\
3 & $\mathrm{CH}_{2} \mathrm{Cl}_{2}$ & 2 & 91 \\
4 & $\mathrm{CHCl}_{3}$ & 4 & 77 \\
5 & $\mathrm{THF}$ & 2 & 88 \\
6 & $\mathrm{DMF}$ & 2 & 90 \\
7 & DMSO & 3 & 87 \\
8 & Toluene & 4 & 41 \\
9 & Benzene & 4 & 37 \\
\hline
\end{tabular}

${ }^{\mathrm{a}}$ Isolated yield 
Table 3. Comparative reaction of compound 4a $(0.010 \mathrm{~mol})$ with 1 -bromo-3-chloropropane $(0.015 \mathrm{~mol})$ in the presence of $\mathrm{TBAB}(0.001 \mathrm{~mol})$ and $\mathrm{Cs}_{2} \mathrm{CO}_{3}(0.010 \mathrm{~mol})$ using solvent-free (microwave, $300 \mathrm{~W}$; thermal, $100^{\circ} \mathrm{C}$ ) versus the solution conditions (MeCN, $30 \mathrm{~mL}$, r.t)

\begin{tabular}{rcc}
\hline Conditions & Time $(\mathrm{min})$ & Yield $^{a}(\%)$ \\
\hline Solution & 120 & 94 \\
Microwave & 6 & 80 \\
Thermal & 120 & 73 \\
\hline
\end{tabular}

${ }^{\mathrm{a}}$ Isolated yield

To investigate the versatility as well as the capacity of our method, the reactions were examined with various $N 1$-substituted nucleobases and alkyl halides (Table 4). As it is shown in Table 4, the reactions proceeded efficiently and the desired N1,N3-dialkylated pyrimidines were obtained in good to excellent yields.

The effect of substituents ( $\mathrm{Me}$ and $\mathrm{F}$ ) on 5-position of 1-alkyl pyrimidines on N3alkylation reaction was also studied. As Table 4 shows, the presence of a fluoro substituent had no significant effect on the reaction but the presence of a methyl substituent lowered the yields and increased the reaction times (Table 4, entries 9-12). Compound 5d was obtained via reaction of carboacyclic nucleoside 4a with ethyl 3-bromopropanoate as well as ethyl acrylate. As Table 4 indicates, the reaction yield was remarkably higher when alkyl halide was used rather than $\alpha, \beta$ unsaturated ester (Table 4, entries 4 and 15). N3-Alkylation of carboacyclic nucleoside $\mathbf{4 a}$ with epoxide in the presence of $\mathrm{TBAB}$ and $\mathrm{Cs}_{2} \mathrm{CO}_{3}$ in $\mathrm{MeCN}$ at room temperature was not successful (Table 4, entry 16).

This method can be easily applied for $N 3$-alkylation of 5'-protected uridine (classic nucleoside) (Table 4, entries 13-14). The 5'-DMT-uridine 6 reacted with 1-bromo-3chloropropane and 2-(4-bromo-butyl)-isoindole-1,3-dione to afford compounds $\mathbf{7 a}$ and $\mathbf{7 b}$ in excellent yields and reasonable times (Table 4, entries 13-14). The sugar residue has a little effect on $N 3$-alkylation of uracil, nevertheless, it was expected that bulky sugar moiety to decrease the $N 3$ tendency for alkylation. 
Table 4. $N 3$-Alkylation of $N 1$-substituted pyrimidine nucleobases with different alkylating agent in the presence of $\mathrm{TBAB}$ and $\mathrm{Cs}_{2} \mathrm{CO}_{3}$ in $\mathrm{MeCN}$ at room temperature

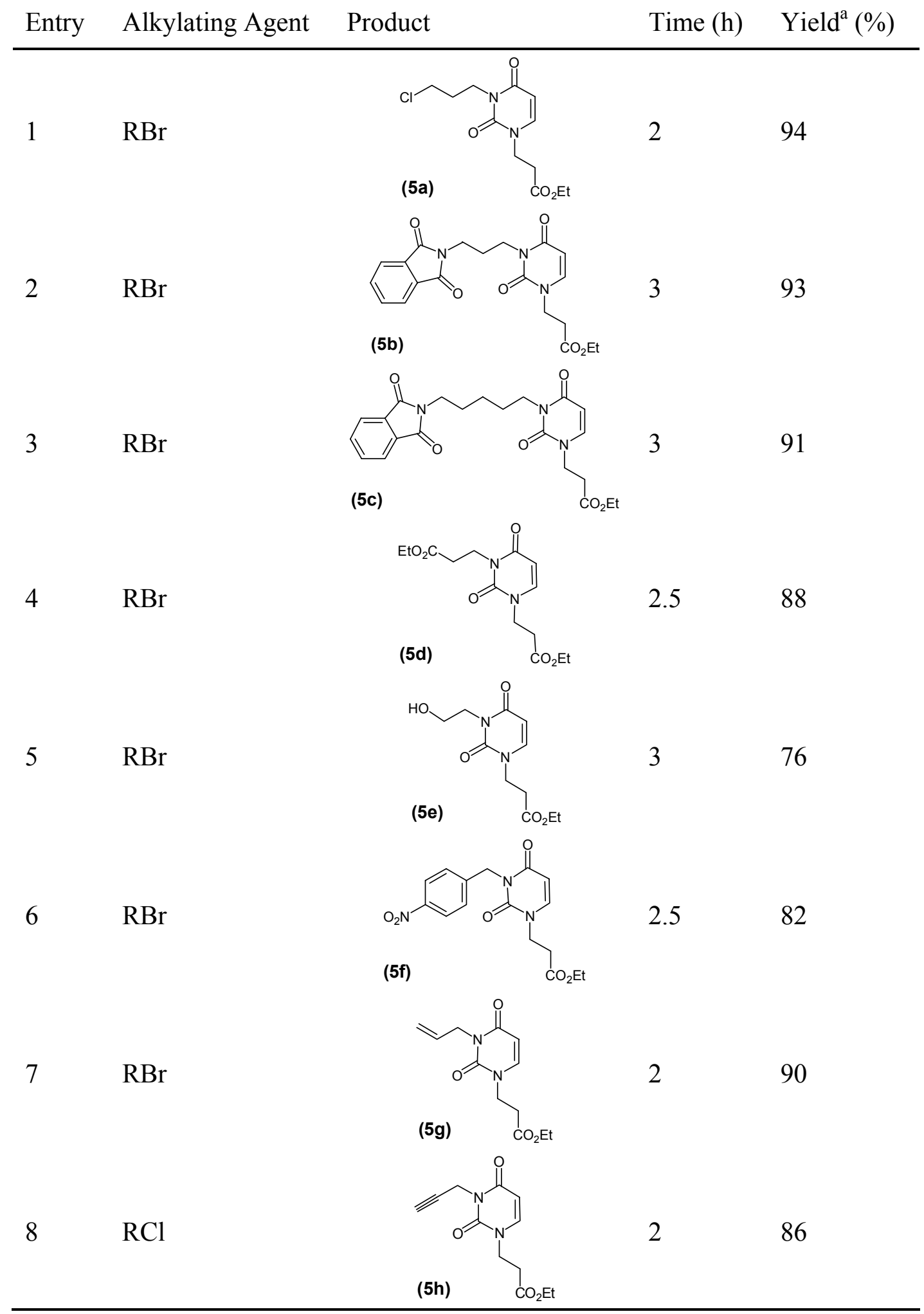


Table 4. N3-Alkylation of $N 1$-substituted pyrimidine nucleobases with different alkylating agent in the presence of $\mathrm{TBAB}$ and $\mathrm{Cs}_{2} \mathrm{CO}_{3}$ in $\mathrm{MeCN}$ at room temperature (continued)

\begin{tabular}{|c|c|c|c|c|}
\hline Entry & Alkylating Agent & Product & Time (h) & Yield $^{\mathrm{a}}(\%)$ \\
\hline 9 & $\mathrm{RBr}$ & & 3 & 79 \\
\hline 10 & $\mathrm{RBr}$ & & 3.5 & 62 \\
\hline 11 & $\mathrm{RBr}$ & & 2 & 93 \\
\hline 12 & $\mathrm{RBr}$ & & 3 & 75 \\
\hline 13 & $\mathrm{RBr}$ & & 12 & 88 \\
\hline 14 & $\mathrm{RBr}$ & & 14 & 87 \\
\hline 15 & $\widehat{\mathrm{CO}_{2} \mathrm{Et}}$ & & 3 & 26 \\
\hline $16^{\mathrm{b}}$ & & & 12 & - \\
\hline
\end{tabular}

${ }^{\mathrm{a}}$ Isolated yield. ${ }^{\mathrm{b}}$ No reaction was observed even by changing the solvent. 


\section{Conclusions}

In summary, we have developed an efficient and simple method for $N 3$-alkylation of $N 1$ alkylpyrimine nucleobases in mild conditions. In this method, 1,3-dialkylpyrimidine nucleobases were synthesized as biologically interesting compounds in good to excellent yields.

\section{Experimental Section}

General Procedures. All chemicals were prepared from Merck or Fluka chemical companies. Solvents were purified and dried according to reported methods and stored over molecular sieves $0.3 \mathrm{~nm} .{ }^{12}$ The progress of reaction was followed with TLC using silica gel SILG/UV 254 plates. Silica gel 60, 0.063-0.200 mm (70-230 mesh ASTM) was used for column chromatography. IR spectra were run on a Shimadzu FTIR-8300 spectrophotometer. The ${ }^{1} \mathrm{H}$ NMR $(250 \mathrm{MHz})$ and ${ }^{13} \mathrm{C}$ NMR (62.5 MHz) were run on a Bruker Avanced DPX-250, FT-NMR spectrometer. Mass spectra were recorded on a Shimadzu GC MS-QP 1000 EX apparatus. Microanalyses were performed on a Perkin-Elmer 240-B microanalyzer.

N3-Alkylation of N1-substituted Pyrimidines with Carbon Electrophiles. A mixture of compounds consisting of 1-alkylpyrimidine nucleobase $(0.010 \mathrm{~mol})$, alkylating agent $(0.015$ $\mathrm{mol})$, TBAB $(0.322 \mathrm{~g}, 0.001 \mathrm{~mol})$ and $\mathrm{Cs}_{2} \mathrm{CO}_{3}(3.258 \mathrm{~g}, 0.010 \mathrm{~mol})$ in anhydrous $\mathrm{MeCN}(30 \mathrm{~mL})$ was stirred at room temperature for appropriate time (Table 3 ). Then, the solids were filtered off and washed with $\mathrm{MeCN}(20 \mathrm{~mL})$. The filtrate and washings were combined, the solvent was evaporated and the residual product was purified by column chromatography on silica gel with EtOAc/ $n$-hexane (1/1).

3-(2,4-Dioxo-3,4-dihydro-2H-pyrimidine-1-yl)-propionic acid ethyl ester (4a). Colourless crystals; yield: $83 \%$; mp $78-80^{\circ} \mathrm{C}$ (Lit. ${ }^{11 \mathrm{a}} \mathrm{mp} 78-80{ }^{\circ} \mathrm{C}$ ).

3-(5-Methyl-2,4-dioxo-3,4-dihydro-2 $H$-pyrimidine-1-yl)-propionic acid ethyl ester (4b). Colourless crystals; yield: $78 \%$; mp $148-149{ }^{\circ} \mathrm{C}$ (Lit. ${ }^{11 a} \mathrm{mp} 148-149^{\circ} \mathrm{C}$ ).

3-(5-Fluoro-2,4-dioxo-3,4-dihydro-2H-pyrimidine-1-yl)-propionic acid ethyl ester (4c). Pale yellow crystals; yield: $81 \%$; mp $122-124{ }^{\circ} \mathrm{C}$ (Lit. ${ }^{11 \mathrm{a}} \mathrm{mp} 122-124{ }^{\circ} \mathrm{C}$ ).

3-[3-(3-Chloro-propyl)-2,4-dioxo-3,4-dihydro-2 $\mathrm{H}$ - pyrimidin-1-yl] propionic acid ethyl ester (5a). Pale yellow oil; isolated yield: $2.71 \mathrm{~g}(94 \%)$; IR (neat) $v_{\max }\left(\mathrm{cm}^{-1}\right)$ : 3055, 2965, 1734, 1705,$1635 ;{ }^{1} \mathrm{H}$ NMR $\left(\mathrm{CDCl}_{3}\right): \delta 1.14\left(3 \mathrm{H}, \mathrm{t}, J=7.1 \mathrm{~Hz}, \mathrm{CH}_{2} \mathrm{CH}_{3}\right), 1.98\left(2 \mathrm{H}, \mathrm{m}, \mathrm{ClCH}_{2} \mathrm{CH}_{2}\right)$, $2.65\left(2 \mathrm{H}, \mathrm{t}, J=5.9 \mathrm{~Hz}, \mathrm{O}=\mathrm{CCCH}_{2}\right), 3.45\left(2 \mathrm{H}, \mathrm{t}, J=7.4 \mathrm{~Hz}, \mathrm{ClCH}_{2} \mathrm{CH}_{2} \mathrm{CH}_{2}\right), 3.87-4.03(6 \mathrm{H}$, complex, $\mathrm{ClCH}_{2}, \mathrm{O}=\mathrm{CCH}_{2} \mathrm{CH}_{2}$ and $\left.\mathrm{CH}_{3} \mathrm{CH}_{2}\right), 5.57\left(1 \mathrm{H}, \mathrm{d}, J=8.0 \mathrm{~Hz}, \mathrm{H}_{5}\right.$ of uracil), $7.27(1 \mathrm{H}, \mathrm{d}$, $J=8.0 \mathrm{~Hz}, \mathrm{H}_{6}$ of uracil); ${ }^{13} \mathrm{C} \mathrm{NMR}\left(\mathrm{CDCl}_{3}\right): \delta 14.4,30.9,33.2,39.2,42.9,46.4,61.3,101.3$, 144.1, 151.6, 163.2, 171.6; MS m/z (\%): 289 (M+1, 15), 288 (M+2), 253 (61), 226 (25), 207 (26), 153 (32), 97 (52), 82 (84), 55 (100); Anal. calcd for $\mathrm{C}_{12} \mathrm{H}_{17} \mathrm{ClN}_{2} \mathrm{O}_{4}$ : C, 49.92; H, 5.93; N, 9.70. Found: C, 50.21; H, 6.10; N. 9.46. 
3-\{3-[3-(1,3-Dioxo-1,3-dihydro-isoindol-2-yl)-propyl]-2,4-dioxo-3,4-dihydro-2H-pyrimidin1-yl\}- propionic acid ethyl ester (5b). Colourless crystals; mp 130-132 ${ }^{\circ} \mathrm{C}$; isolated yield: 3.70 g (93\%); IR (KBr) $v_{\max }\left(\mathrm{cm}^{-1}\right): 3042,2949,1771,1723,1701,1662 ;{ }^{1} \mathrm{H} \mathrm{NMR}\left(\mathrm{CDCl}_{3}\right): \delta 1.19$ $\left(3 \mathrm{H}, \mathrm{t}, J=7.2 \mathrm{~Hz}, \mathrm{CH}_{2} \mathrm{CH}_{3}\right), 2.02(2 \mathrm{H}, \mathrm{m}), 2.73\left(2 \mathrm{H}, \mathrm{t}, J=6.0 \mathrm{~Hz}, \mathrm{O}=\mathrm{CCH}_{2}\right), 3.72(2 \mathrm{H}, \mathrm{t}, J=$ $7.0 \mathrm{~Hz}), 3.92-4.01\left(4 \mathrm{H}\right.$, complex), $4.09\left(2 \mathrm{H}, \mathrm{q}, J=7.2 \mathrm{~Hz}, \mathrm{CH}_{3} \mathrm{CH}_{2}\right), 5.64\left(1 \mathrm{H}, \mathrm{d}, J=8.0 \mathrm{~Hz}, \mathrm{H}_{5}\right.$ of uracil), $7.31\left(1 \mathrm{H}, \mathrm{d}, J=8.0 \mathrm{~Hz}, \mathrm{H}_{6}\right.$ of uracil), $7.67(2 \mathrm{H}, \mathrm{m}), 7.81(2 \mathrm{H}, \mathrm{m}) ;{ }^{13} \mathrm{C} \mathrm{NMR}\left(\mathrm{CDCl}_{3}\right): \delta$ $13.1,25.8,31.9,34.8,37.8,45.1,60.1,100.2$, 122.2, 131.1, 132.9, 142.6, 150.3, 161.9, 167.3, 170.4; MS m/z (\%): $400\left(\mathrm{M}^{+}+1,4\right), 399\left(\mathrm{M}^{+}, 10\right), 354$ (12), 299 (8), 239 (22), 226 (100), 193 (31), 152 (68), 96 (60), 82 (82), 55 (85); Anal. calcd for $\mathrm{C}_{20} \mathrm{H}_{21} \mathrm{~N}_{3} \mathrm{O}_{6}: \mathrm{C}, 60.14 ; \mathrm{H}, 5.30 ; \mathrm{N}$, 10.52. Found: C, 59.95; H, 5.58; N. 10.73 .

\section{3-\{3-[5-(1,3-Dioxo-1,3-dihydro-isoindol-2-yl)-pentyl]-2,4-dioxo-3,4-dihydro-2H-pyrimidin-}

1-yl\}- propionic acid ethyl ester (5c). Colourless crystals; mp 125-127 ${ }^{\circ} \mathrm{C}$; isolated yield: $3.88 \mathrm{~g}$ (91\%); IR (KBr) $v_{\max }\left(\mathrm{cm}^{-1}\right): 3057,2961,1772,1725,1701,1665 ;{ }^{1} \mathrm{H}$ NMR $\left(\mathrm{CDCl}_{3}\right): \delta=1.15$ $\left(3 \mathrm{H}, \mathrm{t}, J=7.0 \mathrm{~Hz}, \mathrm{CH}_{2} \mathrm{CH}_{3}\right), 1.33(2 \mathrm{H}, \mathrm{m}), 1.55-1.66(4 \mathrm{H}$, complex), 2.69 (2H, t, $J=5.9 \mathrm{~Hz}$, $\left.\mathrm{O}=\mathrm{CCH}_{2}\right), 3.60(2 \mathrm{H}, \mathrm{t}, J=6.9 \mathrm{~Hz}),, 3.79-3.90(4 \mathrm{H}$, complex $), 4.08\left(2 \mathrm{H}, \mathrm{q}, J=7.0 \mathrm{~Hz}, \mathrm{CH}_{3} \mathrm{CH}_{2}\right)$, $5.56\left(1 \mathrm{H}, \mathrm{d}, J=7.9 \mathrm{~Hz}, \mathrm{H}_{5}\right.$ of uracil), $7.26\left(1 \mathrm{H}, \mathrm{d}, J=7.9 \mathrm{~Hz}, \mathrm{H}_{6}\right.$ of uracil), $7.64(2 \mathrm{H}, \mathrm{m}), 7.75$ $(2 \mathrm{H}, \mathrm{m}) ;{ }^{13} \mathrm{C} \mathrm{NMR}\left(\mathrm{CDCl}_{3}\right): \delta 14.5,24.5,27.4,28.6,33.4,38.1,41.2,46.5,61.4,101.6,123.5$, 132.5, 134.2, 143.7, 151.7, 163.3, 168.7, 171.7; MS m/z (\%): $428\left(\mathrm{M}^{+}+1,4\right), 427\left(\mathrm{M}^{+}, 8\right), 382$ (8), 267 (28), 213 (59), 160 (72), 113 (63), 97 (55), 82 (85), 554 (100); Anal. calcd for $\mathrm{C}_{22} \mathrm{H}_{25} \mathrm{~N}_{3} \mathrm{O}_{6}$ : C, 61.82; H, 5.90; N, 9.83. Found: C, 62.15; H, 5.66; N. 9.99.

3-[3-(2-Ethoxycarbonyl-ethyl)-2,4-dioxo-3,4-dihydro-2H-pyrimidine-1-yl]-propionic acid ethyl ester (5d). Pale yellow oil; isolated yield: $2.75 \mathrm{~g}\left(88 \%\right.$ ); IR (neat) $v_{\max }\left(\mathrm{cm}^{-1}\right)$ : 3044, 2981, 1732, 1705, 1666; ${ }^{1} \mathrm{H}$ NMR $\left(\mathrm{CDCl}_{3}\right): \delta 1.13\left(6 \mathrm{H}, \mathrm{t}, J=7.1 \mathrm{~Hz}, 2 \mathrm{CH}_{2} \mathrm{CH}_{3}\right), 2.49(2 \mathrm{H}, \mathrm{t}, J=7.5$ $\mathrm{Hz}), 2.65(2 \mathrm{H}, \mathrm{t}, J=6.0 \mathrm{~Hz}), 3.89(2 \mathrm{H}, \mathrm{t}, J=6.0 \mathrm{~Hz}$ ), 3.95-4.08 (6H, complex), $5.57(1 \mathrm{H}, \mathrm{d}, J=$ $7.9 \mathrm{~Hz}, \mathrm{H}_{5}$ of uracil), $7.28\left(1 \mathrm{H}, \mathrm{d}, J=7.9 \mathrm{~Hz}, \mathrm{H}_{6}\right.$ of uracil); ${ }^{13} \mathrm{C} \mathrm{NMR}\left(\mathrm{CDCl}_{3}\right): \delta 14.4,32.4$, 33.2, 37.1, 46.4, 60.8, 61.3, 101.3, 144.1, 151.4, 163.0, 171.3, 171.6; MS m/z (\%): $313\left(\mathrm{M}^{+}+1\right.$, 4), $312\left(\mathrm{M}^{+}, 10\right), 267$ (27), 238 (42), 193 (29), 167 (21), 139 (30), 97 (49), 82 (61), 55 (100); Anal. calcd for $\mathrm{C}_{14} \mathrm{H}_{20} \mathrm{~N}_{2} \mathrm{O}_{6}$ : C, 53.84; H, 6.45; N, 8.97. Found: C, 53.63; H, 6.61; N. 8.80.

3-[3-(2-Hydroxy-ethyl)-2,4-dioxo-3,4-dihydro-2H-pyrimidin-1-yl) propionic acid ethyl ester (5e). Pale yellow oil; isolated yield: $1.95 \mathrm{~g}(76 \%)$; IR (neat) $v_{\max }\left(\mathrm{cm}^{-1}\right): 3438,3079,2969,1731$, 1705, 1651; ${ }^{1} \mathrm{H}$ NMR $\left(\mathrm{CDCl}_{3}\right): \delta 1.17\left(3 \mathrm{H}, \mathrm{t}, J=7.2 \mathrm{~Hz}, \mathrm{CH}_{2} \mathrm{CH}_{3}\right), 2.70(2 \mathrm{H}, \mathrm{t}, J=6.0 \mathrm{~Hz}$, $\left.\mathrm{O}=\mathrm{CCH}_{2}\right), 3.35(1 \mathrm{H}, \mathrm{br}, \mathrm{OH}), 3.73\left(2 \mathrm{H}, \mathrm{t}, J=5.0 \mathrm{~Hz}, \mathrm{HOCH}_{2} \mathrm{CH}_{2}\right), 3.94(2 \mathrm{H}, \mathrm{t}, J=6.0 \mathrm{~Hz}$, $\left.\mathrm{O}=\mathrm{CCH}_{2} \mathrm{CH}_{2}\right), 4.02-4.16\left(4 \mathrm{H}\right.$, complex, $\mathrm{HOCH}_{2}$ and $\left.\mathrm{CH}_{3} \mathrm{CH}_{2}\right), 5.65\left(1 \mathrm{H}, \mathrm{d}, J=7.9 \mathrm{~Hz}, \mathrm{H}_{5}\right.$ of uracil), $7.33\left(1 \mathrm{H}, \mathrm{d}, J=7.9 \mathrm{~Hz}, \mathrm{H}_{6}\right.$ of uracil); ${ }^{13} \mathrm{C} \mathrm{NMR}\left(\mathrm{CDCl}_{3}\right): \delta 14.4,33.2,37.2,43.7,46.5$, 61.4, 101.3, 144.5, 152.1, 164.2, 171.7; MS m/z (\%): $257\left(\mathrm{M}^{+}+1,36\right), 256\left(\mathrm{M}^{+}, 2\right), 213(44), 157$ (19), 139 (17), 97 (55), 82 (100), 55 (75); Anal. calcd for $\mathrm{C}_{11} \mathrm{H}_{16} \mathrm{~N}_{2} \mathrm{O}_{5}$ : C, 51.56; H, 6.29; N, 10.93. Found: C, 51.74; H, 6.07; N. 10.67.

3-[3-(4-Nitrobenzyl)-2,4-dioxo-3,4-dihydro-2H-pyrimidin-1-yl]-propionic acid ethyl ester (5f). Pale yellow crystals, mp 84-86 ${ }^{\circ} \mathrm{C}$; isolated yield: $2.83 \mathrm{~g}(82 \%)$; IR (KBr) $v_{\max }\left(\mathrm{cm}^{-1}\right)$ : 3080 , 2991, 1731, 1713, 1636, 1605, 1491; ${ }^{1} \mathrm{H} \mathrm{NMR}\left(\mathrm{CDCl}_{3}\right): \delta 1.21\left(3 \mathrm{H}, \mathrm{t}, J=7.1 \mathrm{~Hz}, \mathrm{CH}_{2} \mathrm{CH}_{3}\right), 2.74$ 
$\left(2 \mathrm{H}, \mathrm{t}, J=6.1 \mathrm{~Hz}, \mathrm{O}=\mathrm{CCH}_{2}\right), 3.98\left(2 \mathrm{H}, \mathrm{t}, J=6.1 \mathrm{~Hz}, \mathrm{O}=\mathrm{CCH}_{2} \mathrm{CH}_{2}\right), 4.09(2 \mathrm{H}, \mathrm{q}, J=7.1 \mathrm{~Hz}$, $\left.\mathrm{CH}_{3} \mathrm{CH}_{2}\right), 5.15(2 \mathrm{H}, \mathrm{s}, \mathrm{ArCH}), 5.73\left(1 \mathrm{H}, \mathrm{d}, J=8.0 \mathrm{~Hz}, \mathrm{H}_{5}\right.$ of uracil), $7.37\left(1 \mathrm{H}, \mathrm{d}, J=8.0 \mathrm{~Hz}, \mathrm{H}_{5}\right.$

of uracil), $7.58(2 \mathrm{H}, \mathrm{d}, J=8.8 \mathrm{~Hz}), 8.12(2 \mathrm{H}, \mathrm{d}, J=8.8 \mathrm{~Hz}) ;{ }^{13} \mathrm{C} \mathrm{NMR}\left(\mathrm{CDCl}_{3}\right): \delta 14.5,33.3$, 43.9, 46.6, 61.5, 101.5, 123.9, 130.1, 144.1, 144.6, 147.7, 151.7, 163.1, 171.6; MS m/z (\%): 348 $\left(\mathrm{M}^{+}+1,8\right), 347\left(\mathrm{M}^{+}, 36\right), 302$ (14), 273 (15), 170 (40), 124 (13), 97 (56), 82 (61), 55 (100); Anal. calcd for $\mathrm{C}_{16} \mathrm{H}_{17} \mathrm{~N}_{3} \mathrm{O}_{5}$ : C, 55.33; H, 4.93; N, 12.10. Found: C, 55.04; H, 5.31; N. 12.34 .

3-(3-Allyl-2,4-dioxo-3,4-dihydro-2H-pyrimidin-1-yl) propionic acid ethyl ester (5g). Pale yellow oil; isolated yield: $2.27 \mathrm{~g}(90 \%)$; IR (neat) $v_{\max }\left(\mathrm{cm}^{-1}\right): 3048,2978,1735,1701,1640 ;{ }^{1} \mathrm{H}$ $\operatorname{NMR}\left(\mathrm{CDCl}_{3}\right): \delta 1.18\left(3 \mathrm{H}, \mathrm{t}, J=7.1 \mathrm{~Hz}, \mathrm{CH}_{2} \mathrm{CH}_{3}\right), 2.70\left(2 \mathrm{H}, \mathrm{d}, J=5.9 \mathrm{~Hz}, \mathrm{O}=\mathrm{CCH}_{2}\right), 3.93(2 \mathrm{H}$, t, $\left.J=5.9 \mathrm{~Hz}, \mathrm{O}=\mathrm{CCH}_{2} \mathrm{CH}_{2}\right), 4.09\left(2 \mathrm{H}, \mathrm{q}, J=7.1 \mathrm{~Hz}, \mathrm{CH}_{3} \mathrm{CH}_{2}\right), 4.46(2 \mathrm{H}, \mathrm{d}, J=5.8 \mathrm{~Hz}$, $\left.\mathrm{H}_{2} \mathrm{C}=\mathrm{CHCH}_{2}\right), 5.09-5.19\left(2 \mathrm{H}\right.$, complex, $\left.=\mathrm{CH}_{2}\right), 5.62\left(1 \mathrm{H}, \mathrm{d}, J=7.9 \mathrm{~Hz}, \mathrm{H}_{5}\right.$ of uracil), $5.79(1 \mathrm{H}$, $\left.\mathrm{m}, \mathrm{H}_{2} \mathrm{C}=\mathrm{CH}\right), 7.27\left(1 \mathrm{H}, \mathrm{d}, \mathrm{J}=7.9 \mathrm{~Hz}, \mathrm{H}_{6}\right.$ of uracil); ${ }^{13} \mathrm{C} \mathrm{NMR}\left(\mathrm{CDCl}_{3}\right): \delta 14.5,33.4,43.4,46.5$, 61.4, 101.6, 118.1, 131.9, 143.9, 151.5, 163.1, 171.7; MS m/z (\%): $253\left(\mathrm{M}^{+}+1,15\right), 252\left(\mathrm{M}^{+}\right.$, 21), 237 (63), 178 (18), 150 (14), 97 (88), 82 (100), 55 (84); Anal. calcd for $\mathrm{C}_{12} \mathrm{H}_{16} \mathrm{~N}_{2} \mathrm{O}_{4}$ : C, 57.13; H, 6.39; N, 11.10. Found: C, 56.95; H, 6.04; N. 11.43.

3-(2,4-Dioxo-3-prop-2-ynyl-3,4-dihydro-2H-pyrimidin-1-yl) propionic acid ethyl ester (5h). Pale yellow oil; isolated yield: $2.15 \mathrm{~g}$ (86\%); IR (neat) $v_{\max }\left(\mathrm{cm}^{-1}\right): 3248,3071,2980,1735$, 1701, 1636; ${ }^{1} \mathrm{H} \mathrm{NMR}\left(\mathrm{CDCl}_{3}\right): \delta 1.18\left(3 \mathrm{H}, \mathrm{t}, J=7.1 \mathrm{~Hz}, \mathrm{CH}_{2} \mathrm{CH}_{3}\right), 2.14(1 \mathrm{H}, \mathrm{s}), 2.73(2 \mathrm{H}, \mathrm{t}, J=$ $\left.6.1 \mathrm{~Hz}, \mathrm{O}=\mathrm{CCH}_{2}\right), 3.96\left(2 \mathrm{H}, \mathrm{t}, \mathrm{J}=6.1 \mathrm{~Hz}, \mathrm{O}=\mathrm{CCH}_{2} \mathrm{CH}_{2}\right), 4.09\left(2 \mathrm{H}, \mathrm{q}, \mathrm{J}=7.1 \mathrm{~Hz}, \mathrm{CH}_{3} \mathrm{CH}_{2}\right)$, $4.60(2 \mathrm{H}, \mathrm{s}), 5.67\left(1 \mathrm{H}, \mathrm{d}, J=8.0 \mathrm{~Hz}, \mathrm{H}_{5}\right.$ of uracil $), 7.35\left(1 \mathrm{H}, \mathrm{d}, J=8.0 \mathrm{~Hz}, \mathrm{H}_{6}\right.$ of uracil $) ;{ }^{13} \mathrm{C}$ NMR $\left(\mathrm{CDCl}_{3}\right): \delta 14.4,30.5,33.3,38.2,46.5,61.4,71.2,101.3,144.5,151.0,162.3,171.6$; MS m/z (\%): $251\left(\mathrm{M}^{+}+1,19\right), 250\left(\mathrm{M}^{+}, 24\right), 205$ (32), 178 (44), 150 (58), 96 (49), 82 (83), 55 (100); Anal. calcd for $\mathrm{C}_{12} \mathrm{H}_{14} \mathrm{~N}_{2} \mathrm{O}_{4}$ : C, 57.59; H, 5.64; N, 11.19. Found: C, 57.87; H, 5.93; N. 11.50 .

3-[3-(3-Chloro-propyl)-5-methyl-2,4-dioxo-3,4-dihydro-2H-pyrimidin-1-yl] propionic acid ethyl ester (5i). Pale yellow oil; isolated yield: $2.38 \mathrm{~g}$ (79\%); IR (neat) $v_{\max }\left(\mathrm{cm}^{-1}\right)$ : 3061, 2970, 1735, 1699, 1658; ${ }^{1} \mathrm{H} \mathrm{NMR}\left(\mathrm{CDCl}_{3}\right): \delta 1.19\left(3 \mathrm{H}, \mathrm{t}, J=7.1 \mathrm{~Hz}, \mathrm{CH}_{2} \mathrm{CH}_{3}\right), 1.88\left(3 \mathrm{H}, \mathrm{s}, \mathrm{CH}_{3}\right), 2.02$ $\left(2 \mathrm{H}, \mathrm{m}, \mathrm{ClCH}_{2} \mathrm{CH}_{2}\right), 2.69\left(2 \mathrm{H}, \mathrm{t}, J=6.0 \mathrm{~Hz}, \mathrm{O}=\mathrm{CCH}_{2}\right), 3.48\left(2 \mathrm{H}, \mathrm{t}, J=7.3 \mathrm{~Hz}, \mathrm{ClCH}_{2} \mathrm{CH}_{2} \mathrm{CH}_{2}\right)$, 3.96-4.08 (6H, complex, $\mathrm{ClCH}_{2}, \mathrm{O}=\mathrm{CCH}_{2} \mathrm{CH}_{2}$ and $\left.\mathrm{CH}_{3} \mathrm{CH}_{2}\right), 7.22\left(1 \mathrm{H}, \mathrm{s}, \mathrm{H}_{6}\right.$ of thymine); ${ }^{13} \mathrm{C}$ NMR $\left(\mathrm{CDCl}_{3}\right): \delta 14.3,15.2,30.3,33.5,38.4,41.1,45.5,61.2,109.1,135.6,149.2,163.5,171.1$; MS m/z (\%): $303\left(\mathrm{M}^{+}+1,10\right), 302\left(\mathrm{M}^{+}, 4\right), 267$ (54), 225 (33), 180 (13), 156 (27), 97 (67), 55 (100); Anal. calcd for $\mathrm{C}_{13} \mathrm{H}_{19} \mathrm{ClN}_{2} \mathrm{O}_{4}$ : C, 51.57; H, 6.33; N, 9.25. Found: C, 51.39; H, 5.97; N. 9.58 .

3-[3-(2-Hydroxy-ethyl)-5-methyl-2,4-dioxo-3,4-dihydro-2H-pyrimidin-1-yl) propionic acid ethyl ester (5j). Pale yellow oil; isolated yield: $1.68 \mathrm{~g}(62 \%)$; IR (neat) $v_{\max }\left(\mathrm{cm}^{-1}\right): 3045,2963$, 1733, 1693, 1660; ${ }^{1} \mathrm{H}$ NMR $\left(\mathrm{CDCl}_{3}\right): \delta 1.15\left(3 \mathrm{H}, \mathrm{t}, J=7.1 \mathrm{~Hz}, \mathrm{CH}_{2} \mathrm{CH}_{3}\right), 1.90\left(3 \mathrm{H}, \mathrm{s}, \mathrm{CH}_{3}\right)$, $2.72\left(2 \mathrm{H}, \mathrm{t}, J=6.0 \mathrm{~Hz}, \mathrm{O}=\mathrm{CCH}_{2}\right), 3.40(1 \mathrm{H}, \mathrm{br}, \mathrm{OH}), 3.68\left(2 \mathrm{H}, \mathrm{t}, J=5.1, \mathrm{HOCH}_{2} \mathrm{CH}_{2}\right), 3.91$ $\left(2 \mathrm{H}, \mathrm{t}, J=6.0 \mathrm{~Hz}, \mathrm{O}=\mathrm{CCH}_{2} \mathrm{CH}_{2}\right), 4.13-4.22\left(4 \mathrm{H}\right.$, complex, $\mathrm{HOCH}_{2}$ and $\left.\mathrm{CH}_{3} \mathrm{CH}_{2}\right), 7.19(1 \mathrm{H}, \mathrm{s}$, $\mathrm{H}_{6}$ of thymine $){ }^{13} \mathrm{C} \mathrm{NMR}\left(\mathrm{CDCl}_{3}\right): \delta 14.4,15.3,33.9,37.6,42.1,45.3,61.3,108.8,134.2,149.9$, 162.9, 171.8; MS m/z (\%): $271\left(\mathrm{M}^{+}+1,25\right), 270\left(\mathrm{M}^{+}, 7\right), 225$ (37), 169 (24), 124 (10), 97 (88), 
55 (100); Anal. calcd for $\mathrm{C}_{12} \mathrm{H}_{18} \mathrm{~N}_{2} \mathrm{O}_{5}$ : C, 53.33; H, 6.71; N, 10.36. Found: C, 53.65; H, 6.93; N. 10.02 .

3-[3-(3-Chloro-propyl)-5-fluoro-2,4-dioxo-3,4-dihydro-2H-pyrimidin-1-yl] propionic acid ethyl ester (5k). Pale yellow oil; isolated yield: $2.86 \mathrm{~g}(93 \%)$; IR (neat) $v_{\max }\left(\mathrm{cm}^{-1}\right)$ : 3071, 2981, 1729, 1713, 1667; ${ }^{1} \mathrm{H} \mathrm{NMR}\left(\mathrm{CDCl}_{3}\right): \delta 1.20\left(3 \mathrm{H}, \mathrm{t}, J=7.1 \mathrm{~Hz}, \mathrm{CH}_{2} \mathrm{CH}_{3}\right), 2.01(2 \mathrm{H}, \mathrm{m}$, $\left.\mathrm{ClCH}_{2} \mathrm{CH}_{2}\right), 2.79\left(2 \mathrm{H}, \mathrm{t}, J=5.9 \mathrm{~Hz}, \mathrm{O}=\mathrm{CCH}_{2}\right), 3.51\left(2 \mathrm{H}, \mathrm{t}, J=7.2 \mathrm{~Hz}, \mathrm{ClCH}_{2} \mathrm{CH}_{2} \mathrm{CH}_{2}\right), 4.02-$ $4.16\left(6 \mathrm{H}\right.$, complex, $\mathrm{ClCH}_{2}, \mathrm{O}=\mathrm{CCH}_{2} \mathrm{CH}_{2}$ and $\left.\mathrm{CH}_{3} \mathrm{CH}_{2}\right), 7.85\left(1 \mathrm{H}, \mathrm{d}, J=6.5 \mathrm{~Hz}, \mathrm{H}_{6}\right.$ of 5fluorouracil); ${ }^{13} \mathrm{C} \mathrm{NMR}\left(\mathrm{CDCl}_{3}\right): \delta 14.3,30.2,33.8,38.2,40.7,45.5,61.1,119.5,138.7,148.6$, 161.7, 171.2; MS m/z (\%): $307\left(\mathrm{M}^{+}+1,18\right), 306\left(\mathrm{M}^{+}, 6\right), 271$ (68), 229 (42), 205 (12), 128 (19), 97 (81), 55 (100); Anal. calcd for $\mathrm{C}_{12} \mathrm{H}_{16} \mathrm{ClFN}_{2} \mathrm{O}_{4}$ : C, 46.99; H, 5.26; N, 9.13. Found: C, 47.28; H, 4.94; N. 8.85 .

3-[3-(2-Hydroxy-ethyl)-5-fluoro-2,4-dioxo-3,4-dihydro-2H-pyrimidin-1-yl) propionic acid ethyl ester (5l). Pale yellow oil; isolated yield: $2.05 \mathrm{~g}(75 \%)$; IR (neat) $v_{\max }\left(\mathrm{cm}^{-1}\right): 3071,2968$, 1734, 1707, 1657; ${ }^{1} \mathrm{H} \mathrm{NMR}\left(\mathrm{CDCl}_{3}\right): \delta 1.23\left(3 \mathrm{H}, \mathrm{t}, J=7.1 \mathrm{~Hz}, \mathrm{CH}_{2} \mathrm{CH}_{3}\right), 2.81(2 \mathrm{H}, \mathrm{t}, J=5.8 \mathrm{~Hz}$, $\left.\mathrm{O}=\mathrm{CCH}_{2}\right), 3.44(1 \mathrm{H}, \mathrm{br}, \mathrm{OH}), 3.73\left(2 \mathrm{H}, \mathrm{t}, J=5.1 \mathrm{~Hz}, \mathrm{HOCH}_{2} \mathrm{CH}_{2}\right), 3.96(2 \mathrm{H}, \mathrm{t}, J=5.8 \mathrm{~Hz}$, $\left.\mathrm{O}=\mathrm{CCH}_{2} \mathrm{CH}_{2}\right), 4.12-4.23\left(4 \mathrm{H}\right.$, complex, $\mathrm{HOCH}_{2}$ and $\left.\mathrm{CH}_{3} \mathrm{CH}_{2}\right), 7.91\left(1 \mathrm{H}, \mathrm{d}, J=6.5 \mathrm{~Hz}, \mathrm{H}_{6}\right.$ of 5fluorouracil); ${ }^{13} \mathrm{C} \mathrm{NMR}\left(\mathrm{CDCl}_{3}\right): \delta 14.5,34.1,38.9,41.5,45.4,61.3,118.6,139.4,148.3,61.1$, 171.4; MS: $\mathrm{m} / \mathrm{z}(\%)=275\left(\mathrm{M}^{+}+1,13\right), 274\left(\mathrm{M}^{+}, 3\right), 229$ (48), 201 (16), 173 (18), 128 (22), 97 (85), 55 (100); Anal. calcd for $\mathrm{C}_{11} \mathrm{H}_{15} \mathrm{FN}_{2} \mathrm{O}_{5}$ : C, 48.17; H, 5.51; N, 10.21. Found: C, 47.80; H, 5.72; N. 10.49 .

\section{1-\{5-[Bis-(4-methoxy-phenyl)-phenyl-methoxymethyl]-3,4-dihydroxy-tetrahydro-furan-2-} yl\}-3-(3-chloro-propyl)-1 $\boldsymbol{H}$-pyrimidine-2,4-dione (7a). Pale yellow oil; isolated yield: $5.48 \mathrm{~g}$ (88 \%); IR (neat) $v_{\max }\left(\mathrm{cm}^{-1}\right): 3445,3062,2970,1703,1651 ;{ }^{1} \mathrm{H} \mathrm{NMR}\left(\mathrm{CDCl}_{3}\right): \delta 2.05(2 \mathrm{H}, \mathrm{m}$, $\left.\mathrm{ClCH}_{2} \mathrm{CH}_{2}\right), 3.48-3.54$ (4H, complex), $3.75\left(6 \mathrm{H}, \mathrm{s}, 2 \mathrm{OCH}_{3}\right), 4.01\left(2 \mathrm{H}, \mathrm{t}, J=7.0 \mathrm{~Hz}, \mathrm{ClCH}_{2}\right)$, $4.19(1 \mathrm{H}, \mathrm{m}), 4.30(1 \mathrm{H}, \mathrm{m}), 4.44(1 \mathrm{H}, \mathrm{m}), 4.66(2 \mathrm{H}, \mathrm{br}, 2 \mathrm{OH}), 5.46\left(1 \mathrm{H}, \mathrm{d}, J=8.1 \mathrm{~Hz}, \mathrm{H}_{5}\right.$ of uracil), $5.89(1 \mathrm{H}, \mathrm{d}, J=3.0 \mathrm{~Hz}$ ), $6.84(4 \mathrm{H}, \mathrm{d}, J=8.9 \mathrm{~Hz}$ ), 7.20-7.30 (6H, complex), 7.36-7.41 (3H, complex), $7.88\left(1 \mathrm{H}, \mathrm{d}, J=8.1 \mathrm{~Hz}, \mathrm{H}_{6}\right.$ of uracil); ${ }^{13} \mathrm{C} \mathrm{NMR}\left(\mathrm{CDCl}_{3}\right): \delta 30.6,39.1,42.6$, $55.2,62.1,70.1,75.8,83.7,87.0,90.9,101.7,113.3,127.1,128.2,130.1,135.1,135.3,138.2$, 144.3, 151.5, 158.7, 162.7; MS m/z (\%): 588 (M+Cl, 2), 546 (2), 536 (4), 518 (6), 491 (3), 436 (4), 303 (26), 283 (31), 241 (14), 215 (10), 188 (7), 107 (42), 82 (100), 77 (71), 55 (63); Anal. calcd for $\mathrm{C}_{33} \mathrm{H}_{35} \mathrm{~N}_{2} \mathrm{O}_{8}$ : C, 63.61; H, 5.66; N, 4.50. Found: C, 63.87; H, 5.87; N. 4.22.

2-[4-(3-\{5-[Bis-(4-methoxy-phenyl)-phenyl-methoxymethyl]-3,4-dihydroxy-tetrahydrofuran-2-yl\}-2,6-dioxo-3,6-dihydro-2H-pyrimidin-1-yl)-butyl]-isoindole-1,3-dione (7b). IR $(\mathrm{KBr}) v_{\max }\left(\mathrm{cm}^{-1}\right): 3447,3073,2932,1771,1716$, 1651; Pale yellow Crystals; mp 122-124 ${ }^{\circ} \mathrm{C}$; isolated yield: $6.49 \mathrm{~g}(87 \%) ;{ }^{1} \mathrm{H}$ NMR $\left(\mathrm{CDCl}_{3}\right): \delta 1.62-1.73(4 \mathrm{H}$, complex), 3.42-3.50 $(4 \mathrm{H}$, complex), $3.76\left(6 \mathrm{H}, \mathrm{s}, 2 \mathrm{OCH}_{3}\right), 3.91(2 \mathrm{H}, \mathrm{t}, J=7.1 \mathrm{~Hz}),, 4.21(1 \mathrm{H}, \mathrm{m}), 4.31(1 \mathrm{H}, \mathrm{m}), 4.41(1 \mathrm{H}$, $\mathrm{m}), 4.60(2 \mathrm{H}, \mathrm{br}, 2 \mathrm{OH}), 5.44\left(1 \mathrm{H}, \mathrm{d}, J=8.0 \mathrm{~Hz}, \mathrm{H}_{5}\right.$ of uracil), $5.87(1 \mathrm{H}, \mathrm{d}, J=3.0 \mathrm{~Hz}), 6.83(4 \mathrm{H}$, $\mathrm{d}, J=9.0 \mathrm{~Hz}), 7.24-7.29$ (6H, complex), 7.35-7.39 (3H, complex), $7.67(2 \mathrm{H}, \mathrm{m}), 7.80(2 \mathrm{H}, \mathrm{m})$, $7.86\left(1 \mathrm{H}, \mathrm{d}, J=8.1 \mathrm{~Hz}, \mathrm{H}_{6}\right.$ of uracil); ${ }^{13} \mathrm{C} \mathrm{NMR}\left(\mathrm{CDCl}_{3}\right): \delta 24.9,25.9,37.6,40.5,62.2,70.3$, $75.8,83.9,86.9,91.2,101.6,113.2,123.2,127.1,128.0,130.1,132.0,133.9,135.1,135.3,137.9$, 
144.3, 151.5, 158.6, 162.6, 168.5; MS m/z (\%): $587\left(\mathrm{M}^{+}-\mathrm{C}_{9} \mathrm{H}_{6} \mathrm{NO}_{2}, 1\right), 546$ (3), 518 (5), 491 (1), 444 (30), 436 (6), 338 (12), 303 (36), 242 (5), 202 (20), 146 (45), 133 (34), 107 (55), 82 (100), 55 (48); Anal. calcd for $\mathrm{C}_{42} \mathrm{H}_{41} \mathrm{~N}_{3} \mathrm{O}_{10}$ : C, 67.46; H, 5.53; N, 5.62. Found: C, 67.82; H, 5.80; N. 5.89 .

\section{Acknowledgements}

We appreciate Shiraz University and Shiraz University of Technology research councils for financial support of this work. We are also grateful to Prof. H. Sharghi for helpful discussion and to Mr. H. Sajedian Fard for running the NMR spectra.

\section{References and Footnotes}

1. (a) Negwer, M.; Scharnow, H.-G. Organic-Chemical Drugs and their Synonyms, $8^{\text {th }}$ ed.; Wiley-VCH, 2001. (b) Katzung, B. G, Basic clinical pharmacology, $8^{\text {th }}$ edn.; McGraw-Hill, 2001. (c) Kleeman, A.; Engel, J.; Kutscher, B.; Reichert, D. Pharmaceutical Substances, $3^{\text {th }}$ edn.; Stuttgart and New York, 1999. (d) Nair, V.; Chun, B.-K. Arkivoc 2003 (i) 9. (e) Volpini, R.; Costanzi, S.; Lambertucci, C.; Portino, F. R.; Taffi, S.; Vittori, S.; Zablocki, J. A.; Klotz, K.-N.; Cristalli, G. Arkivoc 2004 (v) 301. (f) Tiwari, B. K.; Thakur, A.; Thakur, M.; Pandey, N. D.; Narvi, S. S.; Thakur, S. Arkivoc 2006 (ii) 213. (g) Nawrot, B.; Michalak, O.; Janicka, M.; Maszewska, M.; Wojcik, M.; Nowak, G.; Mikolajczyk, B.; Stec, W. J. Arkivoc 2004 (iii) 151.

2. (a) Gao, H.; Mitra, A. K. Synthesis 2000, 329. (b) Guillarme, S.; Legoupy, S.; Bourgougnon, N.; Aubertin, A.-M.; Huet, F. Tetrahedron 2003, 59, 9635. (c) Guillarme, S.; Legoupy, S.; Aubertin, A.-M.; Olicard, C.; Bourgougnon N.; François Huet, F. Tetrahedron, 2003, 59, 2177. (d) Hernandez, A.-I.; Balzarini, J.; Karlsson, A.; Camarasa, M.-J.; Perez-Perez, M.-J. J. Med. Chem. 2002, 45, 4254. (e) Buck, I. M.; Eleuteri, A.; Reese, C. B. Tetrahedron 1994, $50,9195$.

3. (a) Munter, T.; Cotrell, L.; Stuart, H.; Kronberg, L.; Watson, W. P.; Golding, B. T. Chem. Res. Toxicol. 2002, 15, 1549. (b) De Clercq, E. Advance in Antiviral Drug Design, Vol. 1; Johnsson, N. G., Ed.; Jai: Greenwich, 1993, 18-164. (c) De Clercq, E. Medical Virology 1995, 5, 149. (d) De Clercq, E; Sakuma, T; Baba, M.; Pauwels, R.; Balzarini, J.; Rosenberg, I.; Holy, A. Antiviral Res. 1987, 8, 261. (e) Chu, C. K.; Baker, D. C. Nucleosides and Nucleotides as Antitumor and Antiviral Agents, Eds.; Plenum:, New York, 1993. (f) Burbiel, J. C.; Hockemeyer, J.; Müller, C. E. Arkivoc 2006 (ii) 77. (g) Xia, C.-Q.; Zhu, L.B.; Tan, X.-Y.; Yue, Y.; Yu, X.-Q. Arkivoc 2005 (xv) 81. (h) Salas, M.; Gordillo, B.; González, F. J. Arkivoc 2005 (vi) 172. 
4. (a) Marquez, V. E.; Lim, M.-I. Med. Res. Rev. 1986, 6, 1. (b) Zhu, X.-F. Nucleosides, Nucleotides and Nucleic Acids 2000, 19, 651. (c) Gosselin, G.; Griffe, L.; Meillon, J.-C.; Storer, R. Tetrahedron 2006, 62, 906. (d) Borthwick, A. D.; Biggadike, K. Tetrahedron 1992, 48, 571. (e) Jin, Y. H.; Liu, P.; Wang, J.; Das, U.; Baker, R.; Huggins, J.; Chu, C. K. J. Org. Chem. 2005, 70, 394. (f) Yang, Y.-Y.; Meng, W.-D.; Qing, F.-L. Org. Lett. 2004, 4257. (g) Comin, M. J.; Rodriguez, J. B.; Russ, P.; Marquez, V. E. Tetrahedron 2003, 59, 295. (h) Singha, K.; Achari, B.; Mandal, S. B. Arkivoc 2003 (ix) 75. (i) Corsaro, A.; Chiacchio, U.; Pistarà, V.; Borrello, L.; Romeo, G.; Dalpozzo, R. Arkivoc 2006 (vi) 74. (j) Nair, V.; Jeon, G.-S. Arkivoc 2004 (xiv) 133.

5. (a) Yokoyama, M.; Momotake, A. Synthesis 1999, 1541. (b) Koszytkowska-Stawinska, M.; Sas, W. Tetrahedron Lett. 2004, 45, 5437.

6. (a) Yokoyama, M. Synthesis 2000, 1637. (b) Van Draanen, N. A.; Freeman, G. A.; Short, S. A.; Harvey, R.; Jansen, R.; Szczech, G. W. J. Med. Chem. 1996, 39, 538.

7. (a) Thomas,G. Medicinal Chemisty, John Wiely\& Sons Ltd, 2000. (b) Doerge, R. F, Wilson and Gisvold's textbook of organic medicinal and pharmaceutical chemistry, $8^{\text {th }}$ edn, J. B. Lippincott Company, 1982.

8. Novikov, M. S.; Ozerov, A. A.; Brel, A. K.; Boreko, E. I.; Vladyko, G. V.; Korobchenko, L. V. Khim-Farm. Zh. 1991, 25, 35; Chem. Abstr. 1992, 116, 128848.

9. (a) Hovinen, J. Helv. Chim. Acta 1997, 80, 851. (b) Ogilvie, K. K.; Beaucage, S. L.; Gillen, M. F.; Entwistle, D.; Quilliam, M. Nucleic Acids Research 1979, 6, 1695. (c) Singh, H.; Aggarwal, P.; Kumar, S. Synthesis 1989, 520. (d) Priego, E.-M.; Camarasa, M.-J.; PérezPérez, M.-J. Synthesis 2001, 478.

10. (a) Khalafi-Nezhad, A.; Soltani Rad, M. N.; Khoshnood, A. Synthesis, 2004, 583. (b) Bram, G.; Decodts, G. Synthesis 1985, 543.

11. (a) Khalafi-Nezhad, A.; Zarea, A.; Soltani Rad, M. N.; Mokhtari, B.; Parhami, A. Synthesis, 2005, 419. (b) Khalafi-Nezhad, A.; Mokhtari, B. Tetrahedron Lett. 2004, 45, 6737. (c) Khalafi-Nezhad, A.; Soltani Rad, M. N. ; Hakimelahi, G. H. ; Mokhtari, B. Tetrahedron 2002, 258, 10341.

12. Vogel, A. I. Textbook of Practical Organic Chemistry; $4^{\text {th }}$ ed.; Longman Inc., London and New York, 1978, 264-279. 